\title{
Single-photon pump by Cooper-pair splitting
}

\author{
Mattia Mantovani $\odot,{ }^{1}$ Wolfgang Belzig, ${ }^{1}$ Gianluca Rastelli, ${ }^{1,2}$ and Robert Hussein ${ }^{1}$ \\ ${ }^{1}$ Fachbereich Physik, Universität Konstanz, D-78457 Konstanz, Germany \\ ${ }^{2}$ Zukunftskolleg, Universität Konstanz, D-78457 Konstanz, Germany
}

(Received 23 July 2019; revised manuscript received 24 September 2019; published 13 November 2019)

\begin{abstract}
Hybrid quantum dot-oscillator systems have become attractive platforms to inspect quantum coherence effects at the nanoscale. Here, we investigate a Cooper-pair splitter setup consisting of two quantum dots, each linearly coupled to a local resonator. The latter can be realized either by a microwave cavity or a nanomechanical resonator. Focusing on the subgap regime, we demonstrate that cross-Andreev reflection, through which Cooper pairs are split into both dots, can induce nonlocal correlations between the two resonators. Harnessing these correlations allows to establish and control a nonlocal photon transfer between them. The proposed scheme can act as a photonic valve with single-photon accuracy, with potential applications for quantum heat engines and refrigerators involving mesoscopic resonators.
\end{abstract}

DOI: 10.1103/PhysRevResearch.1.033098

\section{INTRODUCTION}

Nonlocality [1,2] and quantum correlations [3] are at the heart of many quantum technologies [4-6]. In hybrid quantum-dot devices, Cooper pairs are a source of correlated electrons and their nonlocal splitting has experimentally [7-17] and theoretically [18-33] drawn much attention over the last few years. In particular, the nonlocal breaking of the particle-hole symmetry in such Cooper-pair splitters (CPSs) gives rise to peculiar thermoelectric effects [34-37]. However, mesoscopic cavity quantum electrodynamics (cQED) devices [38,39] are excellent tools for correlating few-level systems over a distance [40-45]. Such cQED devices have applications in the readout of charge [46-52], spin [53-57], and valley-orbit states [58,59], as well as few-phonon manipulation when mechanical modes can be cooled close to the ground state [60-65]. A mechanism which induces nonlocal photon or phonon correlations through Cooper-pair transport, implemented in a hybrid setup, bridges the gap between the study of heat flows in quantum-dot-based [34-36,66,67] and circuit-QED devices [68-70].

In this work, we consider a CPS in a double-quantum-dot setup with each dot linearly coupled to a local resonator, constituted by either a microwave cavity [49,51,54,71-74] or a mechanical oscillator [43,75-78]; see Fig. 1(a). We demonstrate that this system is a platform to obtain full control on the heat and photon exchange of two originally uncoupled cavities. This induced coupling arises from the proximity between the dots and the superconducting lead and has a purely nonlocal origin due to cross-Andreev reflection. Subsequent, we discuss the underlying physical mechanism following the lines of Ref. [79], where a single-

Published by the American Physical Society under the terms of the Creative Commons Attribution 4.0 International license. Further distribution of this work must maintain attribution to the author(s) and the published article's title, journal citation, and DOI. quantum-dot system in the single-atom lasing regime has been investigated.

For large intradot Coulomb interactions, $U$, and superconducting gap, $|\Delta| \rightarrow \infty$, the proximity of the superconductor causes a nonlocal splitting (and recombination) of Cooper pairs into both dots with the pairing amplitude $\Gamma_{S}>0$. The corresponding Andreev bound states $| \pm\rangle$ are a coherent superposition of the dots' singlet, $|S\rangle$, and empty state, $|0\rangle$. The dots are further tunnel-coupled to normal contacts, which are largely negative-voltage-biased with respect to the chemical potential $\mu_{S}=0$ of the superconductor. In this configuration, due to single-electron tunneling, the singlet state decays at rate $\Gamma$ into a singly occupied state, $|\alpha \sigma\rangle(\alpha=L, R$ and $\sigma=\uparrow, \downarrow)$, and further into the empty state; see Fig. 1(b). For large dot onsite energies $\epsilon \gtrsim \Gamma_{S}$, the charge hybridization is weak $(|+\rangle \approx|S\rangle,|-\rangle \approx|0\rangle)$, and the transitions $|+\rangle \rightarrow|\alpha \sigma\rangle$ and $|\alpha \sigma\rangle \rightarrow|-\rangle$ are faster than the opposite processes [79]; see Fig. 1(c). This asymmetry in the relaxation ultimately explains how to pump or absorb energy within a single mode, and how to transfer photons between the cavities. In the latter case, when the energy splitting $\delta$ between the Andreev bound states is close to the difference of the cavity frequencies, the relevant level structure of the uncoupled system is summarized in Fig. 1(d). We show below that the effective interaction couples the states $\left|+, n_{L}-1, n_{R}+1\right\rangle$ and $\left|-, n_{L}, n_{R}\right\rangle$, where $n_{\alpha}$ indicates the Fock number in the resonator $\alpha$. An electron tunneling event favours transitions $|+\rangle \rightarrow|\alpha \sigma\rangle \rightarrow|-\rangle$ conserving the photon number. When the system reaches the state $|-\rangle \approx$ $|0\rangle$, this coherent cycle restarts. When the system is in $|+\rangle$, it can again decay. During each cycle, a boson is effectively transferred from the left to the right cavity. Since the two cavities are not isolated, but naturally coupled to external baths, a steady heat flow is eventually established between the cavities.

The effect discussed above refers to a single operation point of the system. More generally, using a master equation approach, we show that the interaction between the CPS and the two resonators opens a rich set of inelastic resonant channels for the electron current through the dots, involving either absorption or emission of photons from a local cavity 
(a)

$L$
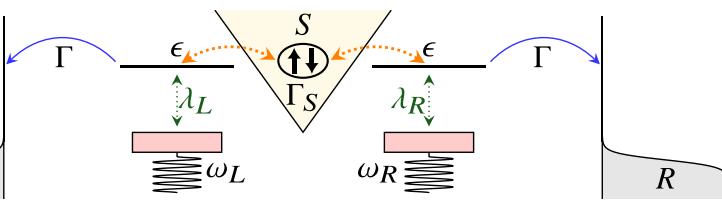

(b)

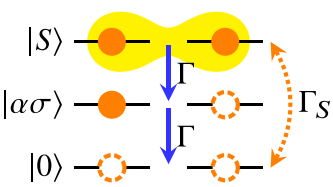

(d)

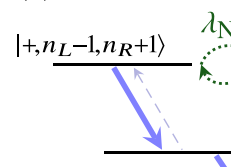

$\left.\delta \approx \omega_{L}-\omega_{R}\right)$

(

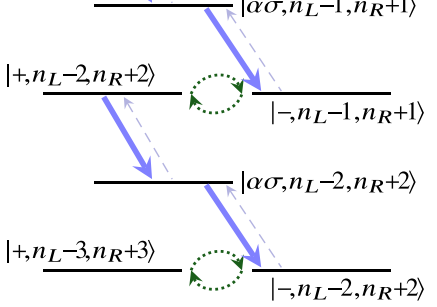

(c)

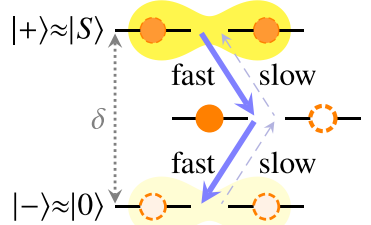

FIG. 1. (a) Cooper-pair splitter consisting of two quantum dots coupled to a common superconductor $(S)$ and two normal-metal contacts $(\alpha=L, R)$. Each dot is capacitively coupled to a local resonator with frequency $\omega_{\alpha}$. (b) At large bias voltage, incoherent tunneling events at rate $\Gamma$ lead to a decay of the singlet state, $|S\rangle$, via a singly occupied one, $|\alpha \sigma\rangle(\sigma=\uparrow, \downarrow)$, to the empty state, $|0\rangle$, whereby $|0\rangle$ and $|S\rangle$ are coherently coupled with amplitude $\Gamma_{S}$. (c) The latter coupling leads to the formation of hybridized $| \pm\rangle$ states of energy splitting $\delta$. For weakly hybridized states $|0\rangle$ and $|S\rangle$, the transitions $| \pm\rangle \leftrightarrow|\alpha \sigma\rangle$ are strongly asymmetric. (d) Photon transfer cycle occurring around the resonance, $\delta \approx \omega_{L}-\omega_{R}$, with the effective coupling strength $\lambda_{\mathrm{NL}}$.

or nonlocal transfer processes. By tuning $\epsilon$ to match these resonances, the CPS acts as a switch allowing the manipulation of heat between the resonators. Each resonant process can be captured with good approximation by an effective Hamiltonian which is valid close to the resonance and generalizes the mechanism described above.

This work is structured as follows. After introducing our model and the employed master equation in Sec. II, we provide therein an effective Hamiltonian describing local and nonlocal transport processes. In Sec. III, we discuss the possibility of simultaneous cooling (and heating) of the resonators. Section IV is dedicated to the nonlocal photon transfer between them, and in Sec. V we analyze the efficiency of this transfer. Finally, we draw our conclusions in Sec. VI.

\section{COOPER-PAIR SPLITTER COUPLED TO RESONATORS}

We consider the effective model for two single-level quantum dots proximized by a $s$-wave superconductor, and each linearly coupled to a local harmonic oscillator. For large intradot Coulomb interaction, $U \gg|\epsilon|$, the subgap physics of the system is described by the effective Hamiltonian [28,32,80-86]

$$
\begin{aligned}
H= & \sum_{\alpha \sigma} \epsilon N_{\alpha \sigma}-\frac{\Gamma_{S}}{2}\left(d_{R \uparrow}^{\dagger} d_{L \downarrow}^{\dagger}-d_{R \downarrow}^{\dagger} d_{L \uparrow}^{\dagger}+\text { H.c. }\right) \\
& +\sum_{\alpha} \omega_{\alpha} b_{\alpha}^{\dagger} b_{\alpha}+\sum_{\alpha, \sigma} \lambda_{\alpha}\left(b_{\alpha}+b_{\alpha}^{\dagger}\right) N_{\alpha \sigma},
\end{aligned}
$$

where $\hbar=1$. Here, $d_{\alpha \sigma}$ is the fermionic annihilation operator for a spin- $\sigma$ electron in $\operatorname{dot} \alpha$, with the corresponding number operator $N_{\alpha \sigma}$ and onsite energy $\epsilon$. The interaction of the dot with the $\alpha$-oscillator of frequency $\omega_{\alpha}$ and corresponding bosonic field $b_{\alpha}$ is realized through the charge term, with coupling constant $\lambda_{\alpha}$. The relevant subspace of the electronic subsystem is spanned by six states: The empty state $|0\rangle$, the four singly occupied states $|\alpha \sigma\rangle=d_{\alpha \sigma}^{\dagger}|0\rangle$ and the singlet state $|S\rangle=\frac{1}{\sqrt{2}}\left(d_{R \uparrow}^{\dagger} d_{L \downarrow}^{\dagger}-d_{R \downarrow}^{\dagger} d_{L \uparrow}^{\dagger}\right)|0\rangle$. Triplet states and doubly occupied states are inaccessible due to large negative voltages, see Fig. 1(a), and large intradot Coulomb repulsion. Finally, in the subgap regime, the superconductor can only pump Cooper pairs, which are in the singlet state. The states $|0\rangle$ and $|S\rangle$ are hybridized due to the $\Gamma_{S}$-term, yielding the Andreev states $|+\rangle=\cos (\theta / 2)|0\rangle+\sin (\theta / 2)|S\rangle$ and $|-\rangle=-\sin (\theta / 2)|0\rangle+$ $\cos (\theta / 2)|S\rangle$, with the mixing angle $\theta=\arctan \left[\Gamma_{S} /(\sqrt{2} \epsilon)\right]$. We denote their energy splitting by $\delta=\sqrt{4 \epsilon^{2}+2 \Gamma_{S}^{2}}$.

Electron tunneling into the normal leads and dissipation for the resonators can be treated in the sequential-tunneling regime to lowest order in perturbation theory, assuming small dot-lead tunneling rates, $\Gamma \ll \Gamma_{S}, k_{B} T$, and large quality factors $Q_{\alpha}=\omega_{\alpha} / \kappa_{\alpha}$ for the resonators, i.e. $\kappa_{\alpha} \ll \omega_{\alpha}, k_{B} T$. Here, $\kappa_{\alpha}$ is the decay rate for the $\alpha$-resonator and $T$ is the temperature of the fermionic and bosonic reservoirs. The fermionic and bosonic transition rates between two eigenstates $|i\rangle$ and $|j\rangle$ of Hamiltonian Eq. (1) are given by Fermi's golden rule [87],

$$
\begin{gathered}
w_{\mathrm{el}, j \leftarrow i}^{\alpha, s}=\Gamma f_{\alpha}^{(s)}\left(s E_{j i}\right) \sum_{\sigma}\left|\left\langle j\left|d_{\alpha \sigma}^{(s)}\right| i\right\rangle\right|^{2}, \\
w_{\mathrm{ph}, j \leftarrow i}^{\alpha, s}=s \kappa_{\alpha} n_{B}\left(E_{j i}\right)\left|\left\langle j\left|b_{\alpha}^{(s)}\right| i\right\rangle\right|^{2},
\end{gathered}
$$

with $f_{\alpha}^{(s)}(x)=\left\{\exp \left[s\left(x-\mu_{\alpha}\right) / k_{B} T\right]+1\right\}^{-1}$ the generalized Fermi function $(s= \pm)$ at chemical potential $\mu_{\alpha}$, and $n_{B}(x)=\left[\exp \left(x / k_{B} T\right)-1\right]^{-1}$ the Bose function. $E_{j i} \equiv E_{j}-E_{i}$ denotes the energy difference between two eigenstates. We use the notation $d_{\alpha \sigma}^{(-)}\left(d_{\alpha \sigma}^{(+)}\right)$for fermionic annihilation (creation) operators, and correspondingly $b_{\alpha}^{( \pm)}$for the bosonic ones. The populations $P_{i}$ of the system eigenstates obey a Pauli-type master equation of the form [28,88,89]

$$
\dot{P}_{i}=\sum_{j} w_{i \leftarrow j} P_{j}-\sum_{j} w_{j \leftarrow i} P_{i},
$$

which admits a stationary solution given by $P_{i}^{\text {st }}$. The total rates entering Eq. (4) are given by $w_{j \leftarrow i}=\sum_{\alpha, s}\left(w_{\mathrm{el}, j \leftarrow i}^{\alpha, s}+\right.$ $\left.w_{\mathrm{ph}, j \leftarrow i}^{\alpha, s}\right)$. As mentioned before, we assume the chemical potentials of the normal leads $\mu_{\alpha}=-e V$ to be largely negativebiased, i.e., $U,|\Delta| \gg e V \gg k_{B} T, \epsilon, \Gamma_{S}$, with $V>0$ and $e>0$ denoting the applied voltage and the electron charge, respectively. In this regime, the electrons flow unidirectionally from the superconductor via the quantum dots into the leads; the temperature of the normal leads becomes irrelevant, and the rates $w_{\mathrm{el}, j \leftarrow i}^{\alpha,+}$ vanish. Under these assumptions, the stationary electron current through lead $\alpha$ is simply given by $I_{\alpha}=e \Gamma \sum_{\sigma}\left\langle N_{\alpha \sigma}\right\rangle$. For a symmetric configuration, as assumed here, both stationary currents coincide, $I_{L}=I_{R}$. To evaluate the stationary current and the other relevant quantities, we diagonalize numerically Hamiltonian Eq. (1), and build the transition rates matrices appearing in Eq. (4). Then, the vector 
of the stationary populations, $P_{i}^{\text {st }}$, is found by setting $\dot{P}_{i}=0$ in Eq. (4) and by solving numerically the resulting linear system.

To explain our numerical results, we perform the LangFirsov polaron transformation [90-92] to Hamiltonian Eq. (1). For an operator $O$, we define the unitary transformation $\bar{O}=$ $e^{\xi} O e^{-\xi}$, with $\xi=\sum_{\alpha \sigma} \Pi_{\alpha} N_{\alpha \sigma}$ and $\Pi_{\alpha}=\left(\lambda_{\alpha} / \omega_{\alpha}\right)\left(b_{\alpha}^{\dagger}-b_{\alpha}\right)$. The polaron-transformed Hamiltonian reads then

$$
\bar{H}=\sum_{\alpha \sigma} \bar{\epsilon}_{\alpha} N_{\alpha \sigma}-\frac{\Gamma_{S}}{\sqrt{2}}\left(|S\rangle\langle 0|X+| 0\rangle\langle S| X^{\dagger}\right)+\sum_{\alpha} \omega_{\alpha} b_{\alpha}^{\dagger} b_{\alpha},
$$

with $\bar{\epsilon}_{\alpha}=\epsilon-\lambda_{\alpha}^{2} / \omega_{\alpha}$ and $X=\exp \left(\sum_{\alpha} \Pi_{\alpha}\right)$ [93]. Equation (5) contains a transverse charge-resonator interaction term to all orders in the couplings $\lambda_{\alpha}$. Intriguingly, this coupling has a purely nonlocal origin stemming from the cross-Andreev reflection. By expanding $X$ in powers of $\Pi \equiv \sum_{\alpha} \Pi_{\alpha}$ assuming small couplings $\lambda_{\alpha} \ll \omega_{\alpha}$, and moving to the interaction picture with respect to the noninteracting Hamiltonian, we can identify a family of resonant conditions given by

$$
\bar{\delta} \approx\left|p \omega_{L} \pm q \omega_{R}\right|,
$$

with $p, q$ nonnegative integers, as discussed in the Appendix. Here, $\bar{\delta}=\sqrt{4 \bar{\epsilon}^{2}+2 \Gamma_{S}^{2}}$ is the renormalized energy splitting of the Andreev states due to the polaron shift, with $\bar{\epsilon}=\epsilon-\sum_{\alpha} \frac{\lambda_{\alpha}^{2}}{2 \omega_{\alpha}}$. The renormalized mixing angle reads $\bar{\theta}=$ $\arctan \left[\Gamma_{S} /(\sqrt{2} \bar{\epsilon})\right]$. Around the conditions stated in Eq. (6), a rotating-wave approximation yields an effective interaction of order $p+q$ in the couplings $\lambda_{\alpha}$. Hereafter, we discuss in detail the resonances at $\bar{\delta}=\omega_{L}=\omega_{R}$ and $\bar{\delta}=\omega_{L}-\omega_{R}$ corresponding to one- and two-photon processes, respectively. They can be fully addressed by expanding $X$ up to second order in $\lambda_{\alpha} / \omega_{\alpha}$ and subsequently performing a rotating-wave approximation; see the Appendix.

\section{SIMULTANEOUS COOLING AND HEATING}

For $\bar{\delta}=\omega_{L}=\omega_{R}$, one can achieve simultaneous cooling as well as heating of both resonators, which is already described by the first order terms in $\lambda_{\alpha}$ of Eq. (5). Here, we consider two identical resonators and tune the dot levels $\epsilon$ around the resonance condition $\bar{\delta} \approx \omega_{\alpha}$, i.e., $\bar{\epsilon}= \pm \sqrt{\omega_{\alpha}^{2}-2 \Gamma_{S}^{2}} / 2$. The effective first-order interaction Hamiltonian reads after a rotating-wave approximation

$$
H_{\mathrm{loc}}=\sum_{\alpha} \frac{1}{2} \lambda_{\alpha} \sin \bar{\theta}\left(b_{\alpha} \tau_{+}+b_{\alpha}^{\dagger} \tau_{-}\right),
$$

as we show in the Appendix. The operators $\tau_{+}=|+\rangle\langle-|$ and $\tau_{-}=|-\rangle\langle+|$describe the hopping between the two-level system formed by the states $|+\rangle$ and $|-\rangle$, coupled to the modes through a transverse Jaynes-Cummings-like interaction. The effective coupling is proportional to $\sin \bar{\theta}=\sqrt{2} \Gamma_{S} / \bar{\delta}$, and, thus, a direct consequence of the nonlocal Andreev reflection. The effective interaction in Eq. (7) coherently mixes the three states $\left|+, n_{L}, n_{R}\right\rangle,\left|-, n_{L}+1, n_{R}\right\rangle$, and $\left|-, n_{L}, n_{R}+1\right\rangle$ which are degenerate for $H_{\text {loc }}=0$. When $|\epsilon| \gtrsim \Gamma_{S}$, the hybridization between the charge states is weak. The sign of $\epsilon$ changes the bare dots' level structure: For $\epsilon\langle 0, \mid+\rangle \approx|0\rangle$ and $|-\rangle \approx|S\rangle$, whereas for $\epsilon>0,|+\rangle \approx|S\rangle$ and $|-\rangle \approx|0\rangle$. In the latter case, the chain of transitions $|+\rangle \rightarrow|\alpha \sigma\rangle \rightarrow|-\rangle$ is faster

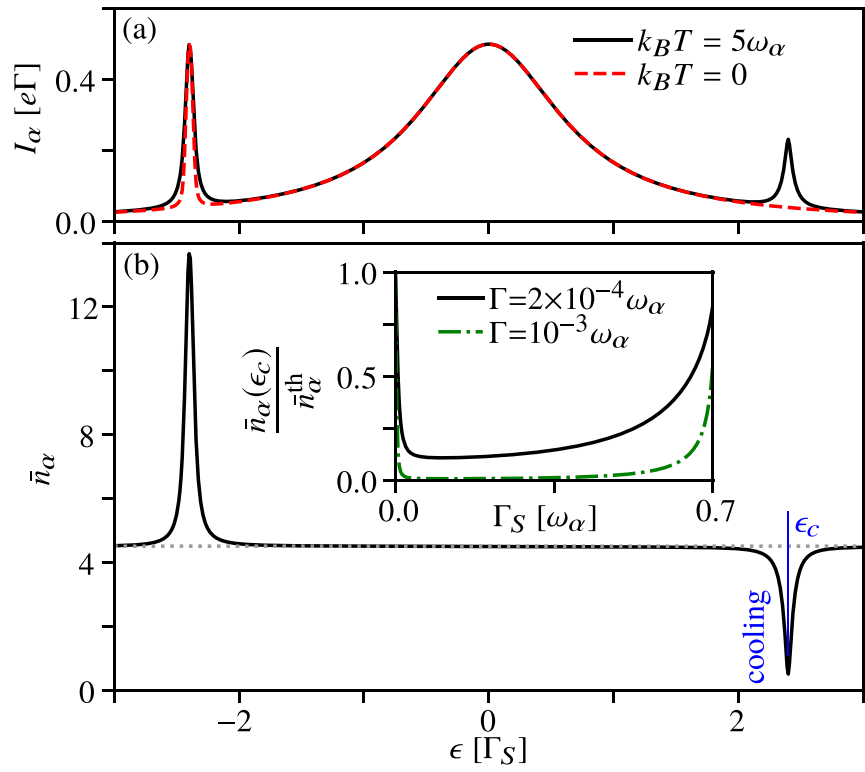

FIG. 2. (a) Current $I_{\alpha}$ for two identical oscillators as a function of the onsite energies $\epsilon$, at zero (dashed line) and finite (solid line) temperature. (b) Average photon occupation $\bar{n}_{\alpha}$ in the $\alpha$-resonator for $k_{B} T=5 \omega_{\alpha}$. The horizontal dotted line corresponds to the thermal occupation. Inset: Photon occupation at $\epsilon=\epsilon_{c}$ as a function of $\Gamma_{S}$, for two different values of $\Gamma$. The curves are rescaled to the thermal occupation value. Other parameters are $\Gamma=2 \times 10^{-4} \omega_{\alpha}, \lambda_{\alpha}=0.02 \omega_{\alpha}$, $Q_{\alpha}=10^{5}, \Gamma_{S}=0.2 \omega_{\alpha}$.

than the opposite process, see Fig. 1(c). For $\epsilon<0$, energy is pumped into the modes. Conversely, for $\epsilon>0$, we can achieve simultaneous cooling of the resonators. In Fig. 2, we show the stationary electron current $I_{\alpha}$ [calculated using the full Hamiltonian Eq. (1)], together with the average photon number $\bar{n}_{\alpha}=\left\langle b_{\alpha}^{\dagger} b_{\alpha}\right\rangle$ of the corresponding resonator, as a function of $\epsilon$. The broad central resonance of width $\Gamma_{S}$ corresponds to the elastic current contribution mediated by the cross-Andreev reflection. The additional inelastic peak at negative $\epsilon$ is related to the emission of photons in both resonators at $\bar{\delta} \approx \omega_{\alpha}$. At finite temperature, a second sideband peak emerges at positive $\epsilon$, where the resonators are simultaneously cooled down. The cavities are efficiently cooled into their ground state for a wide range of values of $\Gamma_{S}$, as can be appreciated in the inset of Fig. 2(b). The optimal cooling region is due to the interplay between the effective interaction with the resonator-which vanishes for small $\Gamma_{S}$ - and the hybridization of the empty and singlet state, which increases as $\epsilon$ approaches the Fermi level of the superconductor and reduces the asymmetry of the transitions $| \pm\rangle \leftrightarrow|\alpha \sigma\rangle$.

\section{NONLOCAL PHOTON TRANSFER}

By keeping terms up to second order in $\lambda_{\alpha}$ in Eq. (5), we can describe the resonances around $\bar{\delta}=\omega_{L}-\omega_{R}$ and $\bar{\delta}=\omega_{L}+\omega_{R}$. Assuming without loss of generality $\omega_{L}>\omega_{R}$, a rotating-wave approximation yields the effective interaction terms $H_{\mathrm{NL}}^{(-)}=\lambda_{\mathrm{NL}}\left(b_{L}^{\dagger} b_{R} \tau_{-}+\right.$H.c. $)$for $\bar{\delta} \approx \omega_{L}-\omega_{R}$, and $H_{\mathrm{NL}}^{(+)}=\lambda_{\mathrm{NL}}\left(b_{L} b_{R} \tau_{+}+\right.$H.c. $)$for $\bar{\delta} \approx \omega_{L}+\omega_{R}$; see the Appendix. These terms show that the two resonators become 

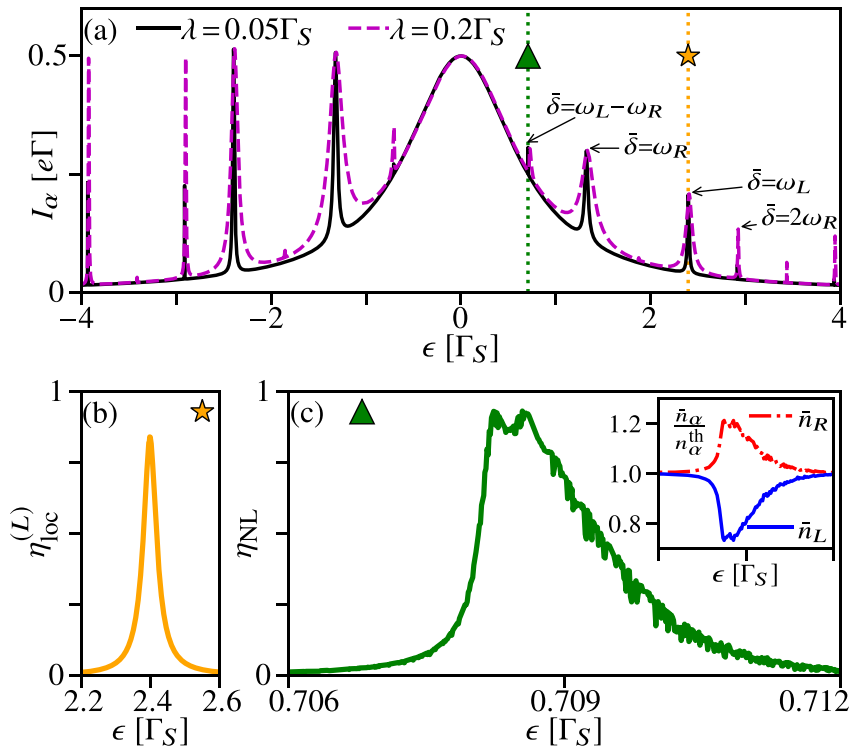

FIG. 3. (a) Current $I_{\alpha}$ through lead $\alpha$ as a function of the onsite energies $\epsilon$, for two different values of $\lambda \equiv \lambda_{L}=\lambda_{R}$. The arrows indicate resonances according to Eq. (6). (b) Local cooling efficiency for the left mode, around $\bar{\delta} \approx \omega_{L}$. (c) Photon transfer efficiency around $\bar{\delta} \approx \omega_{L}-\omega_{R}$. Inset: Average cavity photon number, normalized to the thermal occupation. Parameters are $\Gamma=10^{-4} \Gamma_{S}, \omega_{L}=5 \Gamma_{S}$, $\omega_{R}=3 \Gamma_{S}, Q_{L}=Q_{R}=10^{5}, T=5 \Gamma_{S}$.

indirectly coupled through the charge states, with the strength

$$
\lambda_{\mathrm{NL}}=\frac{\Gamma_{S} \lambda_{L} \lambda_{R}}{\sqrt{2} \omega_{L} \omega_{R}} \cos \bar{\theta} .
$$

We remark that this interaction is, as well, purely nonlocal. $H_{\mathrm{NL}}^{(+)}$describes the hybridization of the states in the subspace $\left|+, n_{L}-1, n_{R}-1\right\rangle$ with $\left|-, n_{L}, n_{R}\right\rangle$, through which photons at different frequencies are simultaneously absorbed (emitted) from (into) both cavities. Conversely, the term $H_{\mathrm{NL}}^{(-)}$describes processes by which the superconductor mediates a coherent transfer of photons between the resonators, by coupling the subspaces $\left|+, n_{L}-1, n_{R}+1\right\rangle$ and $\left|-, n_{L}, n_{R}\right\rangle$; see Fig. 1(d). Notice that this effect vanishes if the two resonators are of the same frequency, as it would require $\bar{\delta}=0$ and, thus, $\Gamma_{S}=0$. In Fig. 3(a), we report the electronic current, again calculated with the full interaction, assuming two different resonator frequencies. In addition to the sideband peaks close to $\bar{\delta}=$ $\omega_{L}$ and $\bar{\delta}=\omega_{R}$, we can identify higher-order multiphoton resonances (e.g., $\bar{\delta}=2 \omega_{R}$, where the cooling cycle involves the absorption of two photons from the same cavity) which can be described in a similar way with a rotating-wave approximation; see the Appendix. Moreover, we observe the secondorder peaks described by $H_{\mathrm{NL}}^{( \pm)}$which are responsible for processes involving both resonators. The inset of Fig. 3(c) reports the average occupation of the resonators in the vicinity of the resonance $\bar{\delta}=\omega_{L}-\omega_{R}$, where the right mode is heated and the left one is cooled. The shape of these resonances differs from the first-order peaks (which are well approximated by Lorentzians): We show in the Appendix how the second-order Hamiltonian contains indeed an additional term proportional to $\sin (\bar{\theta})\left(2 n_{\alpha}+1\right) \tau_{z}$, which causes both a small frequency shift for each resonator (yielding a double-peak structure) and a small renormalization of the splitting $\bar{\delta}$ between the Andreev bound states. Nevertheless, this corrections do not alter the main physics captured by $H_{\mathrm{NL}}^{(-)}$.

\section{HEAT TRANSFER AND EFFICIENCY}

To quantify the performance of both cooling and nonlocal photon transfer, we calculate the stationary heat current $[66,67,87]$

$$
\dot{E}_{\alpha}^{\mathrm{ph}}=\sum_{i, j, s} E_{i j} w_{\mathrm{ph}, j \leftarrow i}^{\alpha, s} P_{i}^{\mathrm{st}},
$$

flowing from the bosonic reservoir $\alpha$ to the corresponding resonator. It is negative (positive) when the resonator is cooled (heated), and vanishes for an oscillator in thermal equilibrium. As a figure of merit for local cooling, we can estimate the number of bosonic quanta subtracted from the resonator on average per unit time, and we can compare it to the rate at which Cooper pairs are injected into the system. The latter rate is given by $\left|I_{S}\right| / 2 e$ with $I_{S}=-\left(I_{L}+I_{R}\right)$ being the Andreev current through the superconductor found from current conservation. Consequently, the local cooling efficiency around $\bar{\delta}=\omega_{\alpha}$ can be defined as $\eta_{\mathrm{loc}}^{(\alpha)}=\frac{2 e\left|\dot{E}_{\alpha}\right|}{\left|I_{s}\right| \omega_{\alpha}}$. Similarly, around $\bar{\delta}=$ $\omega_{L}-\omega_{R}$, we define the heat transfer efficiency

$$
\eta_{\mathrm{NL}}=\frac{2 e\left|\dot{E}_{L}-\dot{E}_{R}\right|}{\left|I_{S}\right|\left(\omega_{L}-\omega_{R}\right)} .
$$

Figures 3(b) and 3(c) show $\eta_{\mathrm{loc}}^{(L)}$ and $\eta_{\mathrm{NL}}$, respectively, as a function of $\epsilon$ close to the corresponding resonances. In both cases, we obtain high efficiencies close to 90\%: Approximately one photon is absorbed from each cavity (local cooling) or transferred from the left to the right cavity (nonlocal transfer) per Cooper pair. The efficiency is essentially limited by two factors: (i) an elastic contribution to the current [the broad resonance of linewidth $\propto \Gamma_{S}$ in Figs. 2(a) and 3(a)] where electrons flow without exchanging energy with the cavities; (ii) a finite fraction of the injected electrons acting against the dominant process (cooling or photon transfer), as illustrated by the dashed blue arrows in Fig. 1(d). Both processes augment with increasing $\Gamma_{S}$ and are a byproduct of the finite hybridization between the empty and the singlet state which, however, is crucial for achieving a nonzero efficiency.

\section{CONCLUSIONS}

We have analyzed a CPS in a double-quantum-dot setup, with local charge couplings to two resonators. We have demonstrated that Cooper-pair splitting can generate a nonlocal transfer of photons and heat from one oscillator to the other, resulting in a stationary energy flow. Such energy flows can also be channeled to cool or heat locally a single cavity. Hence, our system constitutes a versatile tool to fully inspect heat exchange mechanisms in hybrid systems, and is a testbed for quantum thermodynamics investigations involving both electronic and bosonic degrees of freedom. Due to the single-photon nature of the coherent interactions, this can also be extended to achieve few-phonon control and manipulation [94,95], e.g., by implementing time-dependent protocols for the dots' gate voltages to tune dynamically the strength of 
the nonlocal features. Further practical applications include high-efficiency nanoscale heat pumps and cooling devices for nanoresonators.

A discussion on the experimental feasibility of our setup is in order. For single quantum dots coupled to microwave resonators, $\lambda_{\alpha} /(2 \pi)$ can reach $100 \mathrm{MHz}$, with resonators of quality factors $Q \sim 10^{4}$ and frequencies $\omega_{\alpha} /(2 \pi) \sim 7 \mathrm{GHz}$ [49,51]. For mechanical resonators, coupling strengths of $\lambda_{\alpha} /(2 \pi) \sim 100 \mathrm{kHz}$ for frequencies of order $\omega_{\alpha} /(2 \pi) \sim$ $1 \mathrm{MHz}$ and larger quality factors up to $10^{5}-10^{6}$ have been reported [76]. In a double-quantum-dot Cooper-pair splitter setup, the cross-Andreev reflection rate is approximately $\Gamma_{S} \lesssim$ $\sqrt{\Gamma_{S L} \Gamma_{S R}}$, when the distance between the dots is much shorter than the coherence length in the superconducting contact [36]. Here, $\Gamma_{S \alpha}$ is the local Andreev reflection rate which can reach several tens of $\mu \mathrm{eV}$, becoming comparable to the typical microwave resonator frequencies (thus allowing $\Gamma_{S} \lesssim \omega_{\alpha}$ ) while being order of magnitudes lower than the superconducting gap $\Delta$ [96]. Therefore, the regime of parameters we considered lies within the range of state-of-the-art technological capabilities. Moreover, experiments involving Cooper-pair splitters [7-17] or mesoscopic cQED devices with microwave cavities [40,42,46,51,54,72-74] and mechanical resonators $[43,75,76,78]$ are of appealing and growing interest, and therefore promising candidates for the implementation of the system described here.

\section{ACKNOWLEDGMENTS}

This research was supported by the German Excellence Initiative through the Zukunftskolleg and by the Deutsche Forschungsgemeinschaft through Collaborative Research Center SFB 767. R.H. acknowledges financial support from the Carl-Zeiss-Stiftung.

\section{APPENDIX A: POLARON-TRANSFORMED HAMILTONIAN AND EFFECTIVE NONLOCAL INTERACTION}

We report here the derivation of the effective interactions that explain the local cooling or heating, and the nonlocal photon transfer mechanisms. The starting point is the polarontransformed Hamiltonian given in Eq. (5) of the main text.

For small coupling strengths $\lambda_{\alpha} \ll \omega_{\alpha}$, we expand the operators $X$ and $X^{\dagger}$ up to second order in $\lambda_{\alpha}$. The dots-cavities interaction term is

$$
H_{\text {int }}=-\frac{\Gamma_{S}}{\sqrt{2}}\left[i \sigma_{y} \Pi+\sigma_{x}\left(1+\frac{\Pi^{2}}{2}\right)\right]+\mathcal{O}\left(\Pi^{3}\right),
$$

with $i \Pi=\sum_{\alpha} i \Pi_{\alpha}$ the generalized total momentum, $\sigma_{x}=$ $|0\rangle\langle S|+$ H.c. and $\sigma_{y}=-i|0\rangle\langle S|+$ H.c. The $\sigma_{x}$ term describes tunneling between the empty and the singlet state due to the superconductor, and is already present in Hamiltonian Eq. (1) of the main text. Diagonalizing the bare electronic part leads to the hybridized charge states

$$
\begin{gathered}
|+\rangle=\cos \left(\frac{\bar{\theta}}{2}\right)|0\rangle+\sin \left(\frac{\bar{\theta}}{2}\right)|S\rangle, \\
|-\rangle=-\sin \left(\frac{\bar{\theta}}{2}\right)|0\rangle+\cos \left(\frac{\bar{\theta}}{2}\right)|S\rangle,
\end{gathered}
$$

with the mixing angle $\bar{\theta}$ and the energy splitting $\bar{\delta}$ defined in the main text. By introducing the Pauli matrices $\tau_{x}=\tau_{+}+\tau_{-}$, $\tau_{y}=-i\left(\tau_{+}-\tau_{-}\right), \tau_{z}=\left[\tau_{+}, \tau_{-}\right]$with $\tau_{+}=|+\rangle\langle-|$and $\tau_{-}=$ $|-\rangle\langle+|$, we can express the Hamiltonian Eq. (5) of the main text to second order by

$$
\begin{aligned}
\bar{H}= & \sum_{\alpha \sigma} \bar{\epsilon}_{\alpha} N_{\alpha \sigma}+\frac{\bar{\delta}}{2} \tau_{z}+\sum_{\alpha} \omega_{\alpha} b_{\alpha}^{\dagger} b_{\alpha} \\
& -\frac{\Gamma_{S}}{2 \sqrt{2}}\left[2 i \tau_{y} \Pi+\left(\sin \bar{\theta} \tau_{z}+\cos \bar{\theta} \tau_{x}\right) \Pi^{2}\right]+\mathcal{O}\left(\Pi^{3}\right) .
\end{aligned}
$$

We now move to the interaction picture with respect to the noninteracting Hamiltonian $H_{0}=\sum_{\alpha \sigma} \bar{\epsilon}_{\alpha} N_{\alpha \sigma}+\frac{\bar{\delta}}{2} \tau_{z}+$ $\sum_{\alpha} \omega_{\alpha} b_{\alpha}^{\dagger} b_{\alpha}$. By recalling the definition of $\Pi$, we obtain in the interaction picture the Hamiltonian

$$
\begin{aligned}
H_{\mathrm{int}}(t) & \\
= & -\sum_{\alpha} \frac{\lambda_{\alpha} \Gamma_{S}}{\omega_{\alpha} \sqrt{2}}\left(e^{i \omega_{\alpha} t} b_{\alpha}^{\dagger}-e^{-i \omega_{\alpha} t} b_{\alpha}\right)\left(e^{i \bar{\delta} t} \tau_{+}-e^{-i \bar{\delta} t} \tau_{-}\right) \\
& -\frac{\Gamma_{S} \lambda_{L} \lambda_{R}}{\sqrt{2} \omega_{L} \omega_{R}}\left[e^{i \Omega t} b_{L}^{\dagger} b_{R}^{\dagger}+e^{-i \Omega t} b_{L} b_{R}-e^{i(\Delta \omega) t} b_{L}^{\dagger} b_{R}\right. \\
& \left.-e^{-i(\Delta \omega) t} b_{L} b_{R}^{\dagger}\right]\left[\sin (\bar{\theta}) \tau_{z}+\cos (\bar{\theta})\left(e^{i \bar{\delta} t} \tau_{+}+e^{-i \bar{\delta} t} \tau_{-}\right)\right] \\
& -\sum_{\alpha} \frac{\Gamma_{S} \lambda_{\alpha}^{2}}{2 \sqrt{2} \omega_{\alpha}^{2}}\left[e^{2 i \omega_{\alpha} t}\left(b_{\alpha}^{\dagger}\right)^{2}+e^{-2 i \omega_{\alpha} t} b_{\alpha}^{2}-2 b_{\alpha}^{\dagger} b_{\alpha}-1\right] \\
& \times\left[\sin (\bar{\theta}) \tau_{z}+\cos (\bar{\theta})\left(e^{i \bar{\delta} t} \tau_{+}+e^{-i \bar{\delta} t} \tau_{-}\right)\right]+\mathcal{O}\left(\lambda_{\alpha}^{3} / \omega_{\alpha}^{3}\right) .
\end{aligned}
$$

Here, we have introduced $\Omega=\omega_{L}+\omega_{R}$ and $\Delta \omega=\omega_{L}-$ $\omega_{R}$. Hamiltonian Eq. (A5) contains all the terms that lead to cooling, heating, and nonlocal photon transfer. To isolate these features, we will focus on the relevant resonances $\bar{\delta} \approx \omega_{\alpha}, \bar{\delta} \approx$ $\Omega$, and $\bar{\delta} \approx \Delta \omega$.

First, let us consider two identical resonators of frequency $\omega_{\alpha}=\omega$ and tune $\epsilon$ such that $\bar{\delta}=\omega$. Notice that this can be fulfilled by two values of $\epsilon$, of opposite sign. In the following, we restrict Eq. (A5) to first order in $\lambda_{\alpha}$, and then discard the fast-oscillating terms by performing a standard rotating-wave approximation (RWA). Thus, we obtain the time-independent interaction Hamiltonian given by Eq. (7) in the main text,

$$
H_{\mathrm{RWA}}^{\bar{\delta}=\omega}=\sum_{\alpha} \frac{1}{2} \lambda_{\alpha} \sin (\bar{\theta})\left(b_{\alpha} \tau_{+}+b_{\alpha}^{\dagger} \tau_{-}\right) .
$$

We have used here the resonance condition $\omega=\bar{\delta}$ and the relation $\sin \bar{\theta}=\sqrt{2} \Gamma_{S} / \bar{\delta}$.

Let us now consider the nonlocal resonance, $\bar{\delta}=\Delta \omega$. A peculiarity is here, that we have to go to second order in $\lambda_{\alpha}$, since the first-order terms become in the RWA fast rotating and, thus, average to zero. The corresponding effective Hamiltonian reads

$$
\begin{aligned}
H_{\mathrm{RWA}}^{\bar{\delta}=\Delta \omega}= & \sum_{\alpha} \frac{\Gamma_{S} \lambda_{\alpha}^{2}}{2 \sqrt{2} \omega_{\alpha}^{2}}\left(2 n_{\alpha}+1\right) \sin \bar{\theta} \tau_{z} \\
& +\lambda_{\mathrm{NL}}\left(b_{L}^{\dagger} b_{R} \tau_{-}+\text {H.c. }\right),
\end{aligned}
$$


with $n_{\alpha}=b_{\alpha}^{\dagger} b_{\alpha}$ the photon number operator, and $\lambda_{\mathrm{NL}}$ stated in Eq. (8) of the main text. The second term corresponds to the interaction $H_{\mathrm{NL}}^{(-)}$(main text), and is responsible for the coherent transfer of photons between the cavities, leading to a stationary energy flow. The first term in Eq. (A7) proportional to $n_{\alpha} \tau_{z}$ can be seen as a dispersive shift of the cavity frequencies, which depends on the Andreev bound state. As the quantities reported in Fig. 3 of the main text are averages calculated from the density matrix, this translates into a fine double-peak structure of the nonlocal resonance, see Fig. 3(c) of the main text. Further, the additional term proportional to $\tau_{z}$ renormalizes the level splitting $\bar{\delta}$ and, therewith, the resonance condition, $\bar{\delta}=\Delta \omega$.

Considering the condition $\bar{\delta}=\Omega$, we obtain the effective RWA Hamiltonian

$H_{\mathrm{RWA}}^{\bar{\delta}=\Omega}=\sum_{\alpha} \frac{\Gamma_{S} \lambda_{\alpha}^{2}}{2 \sqrt{2} \omega_{\alpha}^{2}}\left(2 n_{\alpha}+1\right) \sin \bar{\theta} \tau_{z}+\lambda_{\mathrm{NL}}\left(b_{L}^{\dagger} b_{R}^{\dagger} \tau_{-}+\right.$H.c. $)$.

Here, the relevant interaction $\left(H_{\mathrm{NL}}^{(+)}\right.$of main text) describes absorption (and emission) from both cavities simultaneously while flipping the Andreev state. So, this second-order effect may entail simultaneous cooling, $\epsilon>0$, and heating, $\epsilon<0$, of both cavities.

From the last line of Eq. (A5), one can infer an effective RWA Hamiltonian governing the resonance condition $\bar{\delta} \approx$ $2 \omega_{\alpha}$. It is similar to Eq. (A6), but involves absorption and emission of two photons from the same cavity. Indeed, this two-photon resonance is also observable in Fig. 3(a) of the main text and yields cavity cooling for $\epsilon>0$ and heating for $\epsilon<0$, respectively.

By including terms up to $n$th order in $\Pi$ in Eq. (A4), one obtains terms $\left(b_{\alpha}\right)^{n}$ and $\left(b_{\alpha}^{\dagger}\right)^{n}$, which, after moving to the interaction picture and performing a suitable RWA, will yield $n$-photon local absorption/emission processes. The expansion contains also terms of the form $\left(b_{\alpha}^{\dagger}\right)^{p}\left(b_{\bar{\alpha}}\right)^{q}$ and $\left(b_{\alpha}^{\dagger}\right)^{p}\left(b_{\bar{\alpha}}^{\dagger}\right)^{q}$ together with their Hermitian conjugates, with $p+q=n$ ( $\bar{\alpha}=R$ if $\alpha=L$ and vice versa). The former terms describe the coherent transfer of $|p-q|$ photons between the cavities, while the latter describes coherent emission and re-absorption of $p$ and $q$ photons from the $\alpha$ and $\bar{\alpha}$ cavity, respectively. The general (approximate) resonance condition thus reads $\bar{\delta} \approx$ $\left|p \omega_{L} \pm q \omega_{R}\right|$, stated in Eq. (6) in the main text. If either $p$ or $q$ is zero, the resonance corresponds to local cooling/heating of the cavities.
[1] H. Buhrman, R. Cleve, S. Massar, and R. de Wolf, Nonlocality and communication complexity, Rev. Mod. Phys. 82, 665 (2010)

[2] N. Brunner, D. Cavalcanti, S. Pironio, V. Scarani, and S. Wehner, Bell nonlocality, Rev. Mod. Phys. 86, 419 (2014).

[3] K. Eckert, J. Schliemann, D. Bruß, and M. Lewenstein, Quantum correlations in systems of indistinguishable particles, Ann. Phys. (NY) 299, 88 (2002).

[4] C. Monroe, Quantum information processing with atoms and photons, Nature 416, 238 (2002).

[5] T. D. Ladd, F. Jelezko, R. Laflamme, Y. Nakamura, C. Monroe, and J. L. O'Brien, Quantum computers, Nature 464, 45 (2010).

[6] C. L. Degen, F. Reinhard, and P. Cappellaro, Quantum sensing, Rev. Mod. Phys. 89, 035002 (2017).

[7] L. Hofstetter, S. Csonka, J. Nygård, and C. Schönenberger, Cooper-pair splitter realized in a two-quantum-dot Y-junction, Nature 461, 960 (2009).

[8] L. G. Herrmann, F. Portier, P. Roche, A. L. Yeyati, T. Kontos, and C. Strunk, Carbon Nanotubes as Cooper-Pair Beam Splitters, Phys. Rev. Lett. 104, 026801 (2010).

[9] L. Hofstetter, S. Csonka, A. Baumgartner, G. Fülöp, S. d'Hollosy, J. Nygård, and C. Schönenberger, Finite-Bias Cooper-Pair Splitting, Phys. Rev. Lett. 107, 136801 (2011).

[10] A. Das, Y. Ronen, M. Heiblum, D. Mahalu, A. V. Kretinin, and H. Shtrikman, High-efficiency Cooper-pair splitting demonstrated by two-particle conductance resonance and positive noise cross-correlation, Nat. Commun. 3, 1165 (2012).

[11] J. Schindele, A. Baumgartner, and C. Schönenberger, NearUnity Cooper-Pair Splitting Efficiency, Phys. Rev. Lett. 109, 157002 (2012).

[12] J. Schindele, A. Baumgartner, R. Maurand, M. Weiss, and C. Schönenberger, Nonlocal spectroscopy of Andreev bound states, Phys. Rev. B 89, 045422 (2014).
[13] G. Fülöp, S. d'Hollosy, A. Baumgartner, P. Makk, V. A. Guzenko, M. H. Madsen, J. Nygård, C. Schönenberger, and S. Csonka, Local electrical tuning of the nonlocal signals in a Cooper-pair splitter, Phys. Rev. B 90, 235412 (2014).

[14] G. Fülöp, F. Domínguez, S. d'Hollosy, A. Baumgartner, P. Makk, M. H. Madsen, V. A. Guzenko, J. Nygård, C. Schönenberger, A. Levy Yeyati, and S. Csonka, Magnetic Field Tuning and Quantum Interference in a Cooper-Pair Splitter, Phys. Rev. Lett. 115, 227003 (2015).

[15] Z. B. Tan, D. Cox, T. Nieminen, P. Lähteenmäki, D. Golubev, G. B. Lesovik, and P. J. Hakonen, Cooper-Pair Splitting by Means of Graphene Quantum Dots, Phys. Rev. Lett. 114, 096602 (2015).

[16] I. V. Borzenets, Y. Shimazaki, G. F. Jones, M. F. Craciun, S. Russo, M. Yamamoto, and S. Tarucha, High efficiency CVD graphene-lead $(\mathrm{Pb})$ Cooper-pair splitter, Sci. Rep. 6, 23051 (2016).

[17] S. Baba, C. Jünger, S. Matsuo, A. Baumgartner, Y. Sato, H. Kamata, K. Li, S. Jeppesen, L. Samuelson, H. Q. Xu, C. Schönenberger, and S. Tarucha, Cooper-pair splitting in two parallel InAs nanowires, New J. Phys. 20, 063021 (2018).

[18] P. Recher, E. V. Sukhorukov, and D. Loss, Andreev tunneling, Coulomb blockade, and resonant transport of nonlocal spinentangled electrons, Phys. Rev. B 63, 165314 (2001).

[19] D. Chevallier, J. Rech, T. Jonckheere, and T. Martin, Current and noise correlations in a double-dot Cooper-pair beam splitter, Phys. Rev. B 83, 125421 (2011).

[20] J. Rech, D. Chevallier, T. Jonckheere, and T. Martin, Current correlations in an interacting Cooper-pair beam splitter, Phys. Rev. B 85, 035419 (2012).

[21] Z. Scherübl, A. Pályi, and S. Csonka, Probing individual split Cooper pairs using the spin qubit toolkit, Phys. Rev. B 89, 205439 (2014). 
[22] P. Trocha and I. Weymann, Spin-resolved Andreev transport through double-quantum-dot Cooper-pair splitters, Phys. Rev. B 91, 235424 (2015).

[23] S. E. Nigg, R. P. Tiwari, S. Walter, and T. L. Schmidt, Detecting nonlocal Cooper-pair entanglement by optical Bell inequality violation, Phys. Rev. B 91, 094516 (2015).

[24] A. Schroer and P. Recher, Detection of nonlocal spin entanglement by light emission from a superconducting $p$ - $n$ junction, Phys. Rev. B 92, 054514 (2015).

[25] B. Probst, F. Domínguez, A. Schroer, A. L. Yeyati, and P. Recher, Signatures of nonlocal Cooper-pair transport and of a singlet-triplet transition in the critical current of a doublequantum-dot Josephson junction, Phys. Rev. B 94, 155445 (2016).

[26] F. Domínguez and A. L. Yeyati, Quantum interference in a Cooper-pair splitter: The three sites model, Phys. E: LowDimens. Syst. Nanostruct. 75, 322 (2016).

[27] E. Amitai, R. P. Tiwari, S. Walter, T. L. Schmidt, and S. E. Nigg, Nonlocal quantum state engineering with the Cooper-pair splitter beyond the Coulomb blockade regime, Phys. Rev. B 93, 075421 (2016).

[28] R. Hussein, L. Jaurigue, M. Governale, and A. Braggio, Double quantum dot Cooper-pair splitter at finite couplings, Phys. Rev. B 94, 235134 (2016).

[29] K. Wrześniewski, P. Trocha, and I. Weymann, Current crosscorrelations in double-quantum-dot-based Cooper-pair splitters with ferromagnetic leads, J. Phys.: Condens. Matter 29, 195302 (2017).

[30] R. Hussein, A. Braggio, and M. Governale, Entanglementsymmetry control in a quantum-dot Cooper-pair splitter, Phys. Status Solidi b 254, 1600603 (2017).

[31] K. Wrześniewski and I. Weymann, Kondo Physics in DoubleQuantum-Dot-Based Cooper-Pair Splitters, Phys. Rev. B 96, 195409 (2017).

[32] N. Walldorf, C. Padurariu, A.-P. Jauho, and C. Flindt, Electron Waiting Times of a Cooper-Pair Splitter, Phys. Rev. Lett. 120, 087701 (2018).

[33] K. Bocian, W. Rudziński, and I. Weymann, Splitting efficiency and interference effects in a Cooper-pair splitter based on a triple quantum dot with ferromagnetic contacts, Phys. Rev. B 97, 195441 (2018).

[34] Z. Cao, T.-F. Fang, L. Li, and H.-G. Luo, Thermoelectricinduced unitary Cooper-pair splitting efficiency, Appl. Phys. Lett. 107, 212601 (2015).

[35] R. Sánchez, P. Burset, and A. L. Yeyati, Cooling by Cooper-pair splitting, Phys. Rev. B 98, 241414(R) (2018).

[36] R. Hussein, M. Governale, S. Kohler, W. Belzig, F. Giazotto, and A. Braggio, Nonlocal thermoelectricity in a Cooper-pair splitter, Phys. Rev. B 99, 075429 (2019).

[37] N. S. Kirsanov, Z. B. Tan, D. S. Golubev, P. J. Hakonen, and G. B. Lesovik, Heat switch and thermoelectric effects based on Cooper-pair splitting and elastic cotunneling, Phys. Rev. B 99, 115127 (2019).

[38] L. Childress, A. S. Sørensen, and M. D. Lukin, Mesoscopic cavity quantum electrodynamics with quantum dots, Phys. Rev. A 69, 042302 (2004).

[39] A. Cottet, M. C. Dartiailh, M. M. Desjardins, T. Cubaynes, L. C. Contamin, M. Delbecq, J. J. Viennot, L. E. Bruhat, B. Douçot, and T. Kontos, Cavity QED with hybrid nanocircuits:
From atomic-like physics to condensed matter phenomena, J. Phys.: Condens. Matter 29, 433002 (2017).

[40] M. Delbecq, L. Bruhat, J. Viennot, S. Datta, A. Cottet, and T. Kontos, Photon-mediated interaction between distant quantum dot circuits, Nat. Commun. 4, 1400 (2013).

[41] N. Lambert, C. Flindt, and F. Nori, Photon-mediated electron transport in hybrid circuit-QED, Europhys. Lett. 103, 17005 (2013).

[42] G. W. Deng, D. Wei, S. X. Li, J. R. Johansson, W. C. Kong, H. O. Li, G. Cao, M. Xiao, G. C. Guo, F. Nori, H. W. Jiang, and G. P. Guo, Coupling two distant double quantum dots with a microwave resonator, Nano Lett. 15, 6620 (2015).

[43] G.-W. Deng, D. Zhu, X.-H. Wang, C.-L. Zou, J.-T. Wang, H.-O. Li, G. Cao, D. Liu, Y. Li, M. Xiao, G.-C. Guo, K.-L. Jiang, X.-C. Dai, and G.-P. Guo, Strongly coupled nanotube electromechanical resonators, Nano Lett. 16, 5456 (2016).

[44] J. E. Ramírez-Muñoz, J. P. Restrepo Cuartas, and H. VinckPosada, Quantum correlations between two cavity QED systems coupled by a mechanical resonator, Eur. Phys. J. B 91, 268 (2018).

[45] M. Trif and P. Simon, Braiding of Majorana Fermions in a Cavity, Phys. Rev. Lett. 122, 236803 (2019).

[46] T. Frey, P. J. Leek, M. Beck, A. Blais, T. Ihn, K. Ensslin, and A. Wallraff, Dipole Coupling of a Double Quantum Dot to a Microwave Resonator, Phys. Rev. Lett. 108, 046807 (2012).

[47] A. Cottet, T. Kontos, and A. L. Yeyati, Subradiant Split Cooper Pairs, Phys. Rev. Lett. 108, 166803 (2012).

[48] J. J. Viennot, M. R. Delbecq, M. C. Dartiailh, A. Cottet, and T. Kontos, Out-of-equilibrium charge dynamics in a hybrid circuit quantum electrodynamics architecture, Phys. Rev. B 89, 165404 (2014).

[49] L. E. Bruhat, J. J. Viennot, M. C. Dartiailh, M. M. Desjardins, T. Kontos, and A. Cottet, Cavity Photons as a Probe for Charge Relaxation Resistance and Photon Emission in a Quantum Dot Coupled to Normal and Superconducting Continua, Phys. Rev. X 6, 021014 (2016).

[50] J. J. Viennot, M. R. Delbecq, L. E. Bruhat, M. C. Dartiailh, M. M. Desjardins, M. Baillergeau, A. Cottet, and T. Kontos, Towards hybrid circuit quantum electrodynamics with quantum dots, C. R. Phys. 17, 705 (2016).

[51] A. Stockklauser, P. Scarlino, J. V. Koski, S. Gasparinetti, C. K. Andersen, C. Reichl, W. Wegscheider, T. Ihn, K. Ensslin, and A. Wallraff, Strong Coupling Cavity QED with Gate-Defined Double Quantum Dots Enabled by a High Impedance Resonator, Phys. Rev. X 7, 011030 (2017).

[52] G. Burkard, M. J. Gullans, X. Mi, and J. R. Petta, Superconductor-semiconductor hybrid cavity quantum electrodynamics, arXiv: 1905.01155.

[53] K. D. Petersson, L. W. McFaul, M. D. Schroer, M. Jung, J. M. Taylor, A. A. Houck, and J. R. Petta, Circuit quantum electrodynamics with a spin qubit, Nature 490, 380 (2012).

[54] J. J. Viennot, M. C. Dartiailh, A. Cottet, and T. Kontos, Coherent coupling of a single spin to microwave cavity photons, Science 349, 408 (2015).

[55] M. Benito, X. Mi, J. M. Taylor, J. R. Petta, and G. Burkard, Input-output theory for spin-photon coupling in Si double quantum dots, Phys. Rev. B 96, 235434 (2017).

[56] X.-Y. Zhu, T. Tu, A.-L. Guo, Z.-q. Zhou, C.-F. Li, and G.-C. Guo, Dynamics of probing a quantum-dot spin qubit with superconducting resonator photons, Sci. Rep. 8, 15761 (2018). 
[57] X. Mi, M. Benito, S. Putz, D. M. Zajac, J. M. Taylor, G. Burkard, and J. R. Petta, A coherent spin-photon interface in silicon, Nature 555, 599 (2018).

[58] S. Kohler, Dispersive readout: Universal theory beyond the rotating-wave approximation, Phys. Rev. A 98, 023849 (2018).

[59] X. Mi, S. Kohler, and J. R. Petta, Landau-Zener interferometry of valley-orbit states in $\mathrm{Si} / \mathrm{SiGe}$ double quantum dots, Phys. Rev. B 98, 161404(R) (2018).

[60] A. D. O’Connell, M. Hofheinz, M. Ansmann, R. C. Bialczak, M. Lenander, E. Lucero, M. Neeley, D. Sank, H. Wang, M. Weides, J. Wenner, J. M. Martinis, and A. N. Cleland, Quantum ground state and single-phonon control of a mechanical resonator, Nature 464, 697 (2010).

[61] G. Piovano, F. Cavaliere, E. Paladino, and M. Sassetti, Coherent properties of nanoelectromechanical systems, Phys. Rev. B 83, 245311 (2011).

[62] J. D. Teufel, T. Donner, D. Li, J. W. Harlow, M. S. Allman, K. Cicak, A. J. Sirois, J. D. Whittaker, K. W. Lehnert, and R. W. Simmonds, Sideband cooling of micromechanical motion to the quantum ground state, Nature 475, 359 (2011).

[63] P. Stadler, W. Belzig, and G. Rastelli, Ground-State Cooling of a Mechanical Oscillator by Interference in Andreev Reflection, Phys. Rev. Lett. 117, 197202 (2016).

[64] P. Stadler, W. Belzig, and G. Rastelli, Charge-vibration interaction effects in normal-superconductor quantum dots, Phys. Rev. B 96, 045429 (2017).

[65] J. B. Clark, F. Lecocq, R. W. Simmonds, J. Aumentado, and J. D. Teufel, Sideband cooling beyond the quantum backaction limit with squeezed light, Nature 541, 191 (2017).

[66] P. A. Erdman, F. Mazza, R. Bosisio, G. Benenti, R. Fazio, and F. Taddei, Thermoelectric properties of an interacting quantumdot-based heat engine, Phys. Rev. B 95, 245432 (2017).

[67] P. A. Erdman, B. Bhandari, R. Fazio, J. P. Pekola, and F. Taddei, Absorption refrigerators based on Coulomb-coupled single-electron systems, Phys. Rev. B 98, 045433 (2018).

[68] P. P. Hofer, J.-R. Souquet, and A. A. Clerk, Quantum heat engine based on photon-assisted Cooper-pair tunneling, Phys. Rev. B 93, 041418(R) (2016).

[69] A. Ronzani, B. Karimi, J. Senior, Y.-C. Chang, J. T. Peltonen, C. Chen, and J. P. Pekola, Tunable photonic heat transport in a quantum heat valve, Nat. Phys. 14, 991 (2018).

[70] S. Dambach, P. Egetmeyer, J. Ankerhold, and B. Kubala, Quantum thermodynamics with a Josephson-photonics setup, Eur. Phys. J. Spec. Top. 227, 2053 (2019).

[71] A. Stockklauser, V. F. F. Maisi, J. Basset, K. Cujia, C. Reichl, W. Wegscheider, T. Ihn, A. Wallraff, and K. Ensslin, Microwave Emission from Hybridized States in a Semiconductor Charge Qubit, Phys. Rev. Lett. 115, 046802 (2015).

[72] X. Mi, J. V. Cady, D. M. Zajac, P. W. Deelman, and J. R. Petta, Strong coupling of a single electron in silicon to a microwave photon, Science 355, 156 (2017).

[73] Y. Li, S.-X. Li, F. Gao, H.-O. Li, G. Xu, K. Wang, D. Liu, G. Cao, M. Xiao, T. Wang, J.-J. Zhang, G.-C. Guo, and G.-P. Guo, Coupling a germanium hut wire hole quantum dot to a superconducting microwave resonator, Nano Lett. 18, 2091 (2018).

[74] T. Cubaynes, M. R. Delbecq, M. C. Dartiailh, R. Assouly, M. M. Desjardins, L. C. Contamin, L. E. Bruhat, Z. Leghtas,
F. Mallet, A. Cottet, and T. Kontos, Highly coherent spin states in carbon nanotubes coupled to cavity photons, npj Quantum Inf. 5, 47 (2019).

[75] A. Benyamini, A. Hamo, S. V. Kusminskiy, F. von Oppen, and S. Ilani, Real-space tailoring of the electron-phonon coupling in ultraclean nanotube mechanical resonators, Nat. Phys. 10, 151 (2014).

[76] Y. Okazaki, I. Mahboob, K. Onomitsu, S. Sasaki, and H. Yamaguchi, Gate-controlled electromechanical backaction induced by a quantum dot, Nat. Commun. 7, 11132 (2016).

[77] Y. Wen, N. Ares, F. J. Schupp, T. Pei, G. A. D. Briggs, and E. A. Laird, A coherent nanomechanical oscillator driven by single-electron tunnelling, Nat. Phys. (2019), doi: 10.1038/s41567-019-0683-5.

[78] C. Urgell, W. Yang, S. L. de Bonis, C. Samanta, M. J. Esplandiu, Q. Dong, Y. Jin, and A. Bachtold, Cooling and self-oscillation in a nanotube electromechanical resonator, Nat. Phys. (2019), doi: 10.1038/s41567-019-0682-6.

[79] G. Rastelli and M. Governale, Single atom laser in normalsuperconductor quantum dots, Phys. Rev. B 100, 085435 (2019).

[80] A. V. Rozhkov and D. P. Arovas, Interacting-impurity Josephson junction: Variational wave functions and slave-boson meanfield theory, Phys. Rev. B 62, 6687 (2000).

[81] T. Meng, S. Florens, and P. Simon, Self-consistent description of Andreev bound states in Josephson quantum dot devices, Phys. Rev. B 79, 224521 (2009).

[82] J. Eldridge, M. G. Pala, M. Governale, and J. König, Superconducting proximity effect in interacting double-dot systems, Phys. Rev. B 82, 184507 (2010).

[83] A. Braggio, M. Governale, M. G. Pala, and J. König, Superconducting proximity effect in interacting quantum dots revealed by shot noise, Solid State Commun. 151, 155 (2011).

[84] S. Droste, S. Andergassen, and J. Splettstoesser, Josephson current through interacting double quantum dots with spin-orbit coupling, J. Phys.: Condens. Matter 24, 415301 (2012).

[85] B. Sothmann, S. Weiss, M. Governale, and J. König, Unconventional superconductivity in double quantum dots, Phys. Rev. B 90, 220501(R) (2014).

[86] S. Weiss and J. König, Odd-triplet superconductivity in singlelevel quantum dots, Phys. Rev. B 96, 064529 (2017).

[87] G. Benenti, G. Casati, K. Saito, and R. S. Whitney, Fundamental aspects of steady-state conversion of heat to work at the nanoscale, Phys. Rep. 694, 1 (2017).

[88] O. Sauret, D. Feinberg, and T. Martin, Quantum master equations for the superconductor-quantum dot entangler, Phys. Rev. B 70, 245313 (2004).

[89] M. Governale, M. G. Pala, and J. König, Real-time diagrammatic approach to transport through interacting quantum dots with normal and superconducting leads, Phys. Rev. B 77, 134513 (2008).

[90] I. G. Lang and Y. A. Firsov, Kinetic theory of semiconductors with low mobility, Zh. Eksp. Teor. Fiz. 43, 1843 (1962) [Sov. Phys. JETP 16, 1301 (1963)].

[91] T. Brandes and B. Kramer, Spontaneous Emission of Phonons by Coupled Quantum Dots, Phys. Rev. Lett. 83, 3021 (1999).

[92] T. Brandes, Coherent and collective quantum optical effects in mesoscopic systems, Phys. Rep. 408, 315 (2005). 
[93] Notice that the polaron transformation also gives rise to a modification of the tunneling Hamiltonian, which describes inelastic dot-lead tunneling events scaling with $\Gamma\left(\lambda_{\alpha} / \omega_{\alpha}\right)^{2}$. However, in our operative regime, such processes can be neglected since $\Gamma \ll \Gamma_{S}$ and $\lambda_{\alpha} \ll \omega_{\alpha}$ ensure $\Gamma\left(\lambda_{\alpha} / \omega_{\alpha}\right)^{2} \ll \Gamma \ll \Gamma_{S}$.

[94] H. Okamoto, A. Gourgout, C.-Y. Chang, K. Onomitsu, I. Mahboob, E. Y. Chang, and H. Yamaguchi, Coherent phonon manipulation in coupled mechanical resonators, Nat. Phys. 9, 480 (2013).
[95] D. Zhu, X.-H. Wang, W.-C. Kong, G.-W. Deng, J.-T. Wang, H.-O. Li, G. Cao, M. Xiao, K.-L. Jiang, X.-C. Dai, G.-C. Guo, F. Nori, and G.-P. Guo, Coherent phonon Rabi oscillations with a high-frequency carbon nanotube phonon cavity, Nano Lett. 17, 915 (2017).

[96] J. Gramich, A. Baumgartner, and C. Schönenberger, Resonant and Inelastic Andreev Tunneling Observed on a Carbon Nanotube Quantum Dot, Phys. Rev. Lett. 115, 216801 (2015). 